hep-ph/0603154

\title{
Lowering solar mixing angle in inverted hierarchy without charged lepton corrections
}

\author{
N.Nimai Singh ${ }^{a} ! 1$, Monisa Rajkhowa ${ }^{a, b}$ and Abhijit Borah $^{c}$ \\ ${ }^{a}$ Department of Physics, Gauhati University, Guwahati-781 014, India \\ ${ }^{b}$ Department of Physics, Science College, Jorhat-785 010, Assam, India \\ ${ }^{c}$ Department of Physics, Fazl Ali College, Mokokchung - 798 601, Nagaland, \\ India
}

\begin{abstract}
In the present work, the inverted hierarchical neutrino mass model which is characterised by opposite $\mathrm{CP}$ parity in the first two mass eigenvalues $\left(m_{1},-m_{2}, m_{3}\right)$, is studied in order to lower the predicted value of solar mixing angle $\tan ^{2} \theta_{12}$, from the tri-bimaximal mixing (TBM), without sacrificing the conditions of maximal atmospheric mixing angle $\left(\theta_{23}=\pi / 4\right)$ and zero reactor angle $\left(\theta_{13}=0\right)$. The present attempt is different from the earlier approach where the correction from the charged lepton mass matrix is included in the leptonic mixing matrix to lower the prediction on solar mixing angle. The lowering of the solar mixing angle without charged lepton correction, can be obtained through the variation of the input value of a flavour twister term present in the texture of neutrino mass matrix having a 2-3 symmetry. The present analysis agrees with the latest experimental bounds on $\triangle m_{21}^{2}$ and $\left|\triangle m_{23}^{2}\right|$. It also represents an important result on the survival of the inverted hierarchical neutrino mass models having opposite CP parity in the first two eigenvalues.
\end{abstract}

\footnotetext{
${ }^{1}$ Regular Associate, The Abdus Salam ICTP, Trieste, Italy E-mail: nimai03@yahoo.com
} 


\section{Introduction}

Recent observational data [1] on neutrino oscillations, indicates a clear departure from the tri-bimaximal mixing(TBM) or Harrison-Perkins-Scott(HPS) mixing pattern[2]. The most recent SNO experimental determination[3] of solar mixing angle leads to $\tan ^{2} \theta_{12}=0.45_{-0.08}^{+0.09}$ compared with $\tan ^{2} \theta_{12}=0.5$ in HPS scheme. So far there is no strong claim for a clear departure from the maximal atmospheric mixing angle $\left(\tan ^{2} \theta_{23}=1.0\right)$ and zero value of CHOOZ angle $\left(\sin \theta_{13}=0\right)$, though only upper bound for $\sin \theta_{13}$ has been known at the moment. Future measurements may find a very small value of $\sin \theta_{13}$ which can be approximated by zero[4]. Such possibility does not yet contradict with the non-observation of Dirac CP phase angle. Relevance of exact zero value of $\mathrm{CHOOZ}$ mixing angle is gaining momentum in the literature, and there are several discussions [5] on the experimental setup for mass hierarchy measurements under $\sin \theta_{13}=0$ condition.

Since the present data on solar and atmospheric neutrino oscillation experiments give only the mass-square differences, we usually have three models of neutrino mass levels[6], (a) degenerate model: $m_{1} \sim m_{2} \sim m_{3}>>$ $\sqrt{\triangle m_{23}^{2}}$, (b) inverted hierarchical model: $m_{1} \sim m_{2}>>m_{3}$ with $\triangle m_{23}^{2}=$ $m_{3}^{2}-m_{2}^{2}<0$ and $m_{1,2} \sim \sqrt{\triangle m_{23}^{2}}$, and (c) normal hierarchical model: $m_{1}<<m_{2}<<m_{3}$ with $\triangle m_{23}^{2}>0$ and $m_{3} \sim \sqrt{\triangle m_{23}^{2}}$. Relative phase of CP parity between these mass eigenvalues will again lead to further subdivisions in these three categories of neutrino mass models[6].

Inspite of inferences drawn from the presently available neutrino experiments[1], the current scenario concerning the pattern of the three neutrino masses, is not a clear one[7,8], though some reactor-based experiments are trying to measure the correct pattern of neutrino masses[9]. In this context, the inverted hierarchical model having opposite CP parity in first two mass eigenvalues $\left(m_{1},-m_{2}, m_{3}\right)$, has been given some special attention due to its high stability under radiative corrections in the minimal supersymmetric standard model(MSSM)[10-12], and considerable theoretical effort has been made in this direction during the last one decade[13].

The origin of the inverted hierarchical mass model can be routed through the exact global charge $\left(L_{e}-L_{\mu}-L_{\tau}\right)$ symmetry, and its subsequent breaking due to possible quantum gravity corrections or some flavour $U(1)_{F}$ symmetry violation[14]. A general form of inverted hierarchical mass matrix having a 
2-3 symmetry in $\nu_{\mu} \leftrightarrow \nu_{\tau}$ sector, can be written as

$$
m_{L L}=\left(\begin{array}{ccc}
\delta_{1} & 1 & 1 \\
1 & \delta_{2} & \delta_{3} \\
1 & \delta_{3} & \delta_{2}
\end{array}\right) m_{0}
$$

where the perturbation parameters $\delta_{1}, \delta_{2}, \delta_{3}$ are assumed to be smaller than unity, and $m_{0}$ is the input quantity representing neutrino mass scale[11]. Such form of neutrino mass matrix can be successfully generated[15] by using the celebrated see-saw formula.

The conditions of maximal atmospheric mixing $\left(\tan ^{2} \theta_{23}=1\right)$ and $\sin \theta_{13}=0$, are the general consequences of the 2-3 symmetry. However, the prediction on solar mixing angle $\tan ^{2} \theta_{12}$, is nearly maximal $\left(\tan ^{2} \theta_{12} \sim 1\right)$. Several attempts[14-16] have been made to tone down the maximal solar mixing angle, while keeping $\sin \theta_{13}$ within the observational bound. In particular, the correction, $U_{e L}$ from the diagonalization of the charged lepton mass matrix with non-zero texture in 1-2 block, in the leptonic mixing matrix, $U_{M N S}=U_{\nu L}^{\dagger} U_{e L}$, can reduce the solar mixing angle from its maximal value[11]. However, this correction on solar angle is strongly constrained by the observed smallness of $\sin \theta_{13}$, as the amount of deviation from a maximal solar angle is of the order of $\theta_{13}$. The mechanism can only work[5] if the value of $\theta_{13}$ is very close to its present upper bound, $\sin \theta_{13}<0.16$. Since the upper bound of $\sin \theta_{13}$ is known, $\sin \theta_{13}$ may be very "small" or zero exactly[4,5].

In the present work, we propose a particular texture of the inverted hierarchical neutrino mass model, which has the potential to lower the solar mixing angle from its tri-bimaximal value without affecting the maximal atmospheric mixing $\left(\tan ^{2} \theta_{23}=1\right)$ and $\sin \theta_{13}=0$ conditions. In section 2 , we present a parametrisation of neutrino mass matrix in terms of a flavour twister which is important for giving deviation from tribimaximal mixings. Section 3 is devoted to numerical analysis using MATHEMATICA and main results of the investigation. In section 4 we conclude with a summary and discussion. 


\section{Parametrisation in term of flavour twister and deviation from tribimaximal mixings}

We diagonalise the neutrino mass matrix $m_{L L}$ in equation (1). We get the following mass eigenvalues,

$$
\begin{array}{r}
m_{1,2}=\frac{m_{0}}{2}\left[\left(\delta_{1}+\delta_{2}+\delta_{3}\right) \pm x\right], \quad m_{3}=m_{0}\left[\left(\delta_{2}-\delta_{3}\right)\right] ; \\
x^{2}=8+\left(\delta_{1}^{2}+\delta_{2}^{2}+\delta_{3}^{2}\right)-2 \delta_{1} \delta_{2}-2 \delta_{1} \delta_{3}+2 \delta_{2} \delta_{3} .
\end{array}
$$

The three mixing angles are given by

$$
\tan ^{2} \theta_{23}=1, \quad \sin \theta_{13}=0, \quad \tan 2 \theta_{12}=\frac{2 \sqrt{2}}{\left(\delta_{1}-\delta_{2}-\delta_{3}\right)} .
$$

In general, any mass matrix of the type in eqation (1) having 2-3 symmetry, can be diagonalized by the following unitary matrix $\left(\theta_{23}=\pi / 4, \theta_{13}=0\right)[4,17]$,

$$
U=\left(\begin{array}{ccc}
\cos \theta_{12} & -\sin \theta_{12} & 0 \\
\frac{\sin \theta_{12}}{\sqrt{2}} & \frac{\cos \theta_{12}}{\sqrt{2}} & -\frac{1}{\sqrt{2}} \\
\frac{\sin \theta_{12}}{\sqrt{2}} & \frac{\cos \theta_{12}}{\sqrt{2}} & \frac{1}{\sqrt{2}}
\end{array}\right)
$$

where the solar angle $\theta_{12}$ in eq.(4) is arbitrary, but it can be fixed by input values $\delta_{1,2,3}$ appearing in the texture of neutrino mass matrix (1). The tri-bimaximal mixing(TBM) or Harrison-Perkinson-Scott (HPS) mixing pat$\operatorname{tern}[2]$ is thus obtained when we choose $\cos \theta_{12}=\sqrt{\frac{2}{3}}$, and $\sin \theta_{12}=\frac{1}{\sqrt{3}}$, leading to $\tan ^{2} \theta_{12}=\frac{1}{2}$ (or $\tan 2 \theta_{12}=2 \sqrt{2}$ ), and it assumes the following form,

$$
U_{T B M}=\left(\begin{array}{ccc}
\sqrt{\frac{2}{3}} & -\frac{1}{\sqrt{3}} & 0 \\
\frac{1}{\sqrt{6}} & \frac{1}{\sqrt{3}} & -\frac{1}{\sqrt{2}} \\
\frac{1}{\sqrt{6}} & \frac{1}{\sqrt{3}} & \frac{1}{\sqrt{2}}
\end{array}\right)
$$

For diagonal neutrino mass matrix $D=\operatorname{diag}\left(m_{1}, m_{2}, m_{3}\right)$, the mass matrix $m_{L L}=U_{T M B} D U_{T B M}^{\dagger}$ generally takes a simple form,

$$
m_{L L}=\left(\begin{array}{ccc}
A & B & B \\
B & A-C & B+C \\
B & B+C & A-C
\end{array}\right) m_{0}
$$


where the elements are expressible in terms of linear combinations of three masses. This particular form of mass matrix (7) is a consequence of tribimaximal mixings and can also be derived from the general $S_{3}$ symmetry[18]. It puts certain contraints for tribimaximal mixings on the mass matrix of eq.(1). From eq.(4) we have $\left(\delta_{1}-\delta_{2}-\delta_{3}\right)= \pm 1$ for tri-bimaximal mixings, and this condition requires only two parameters in term of a flavour twister[19]. Thus the condition of tribimaximal mixings and deviations from it can be expressed in term of flavour twister $\eta / \epsilon$,

$\left(\delta_{1}-\delta_{2}-\delta_{3}\right)=(2-\eta / \epsilon)$.

This condition can be easily satisfied by the choice of a simple parametrisation of $\delta_{1}, \delta_{2}, \delta_{3}, m_{0}$ in terms of only two new parameters $\eta$ and $\epsilon$,

$$
\delta_{1}=2\left(1-\frac{1}{2 \epsilon}\right), \quad \delta_{2}=-\frac{1}{2 \epsilon}, \quad \delta_{3}=\left(\frac{\eta}{\epsilon}-\frac{1}{2 \epsilon}\right), \quad m_{0}=(0.05 \times \epsilon) \mathrm{eV}
$$

Substituting (8) in eq.(1),

$$
m_{L L}=\left(\begin{array}{ccc}
1-2 \epsilon & -\epsilon & -\epsilon \\
-\epsilon & \frac{1}{2} & \frac{1}{2}-\eta \\
-\epsilon & \frac{1}{2}-\eta & \frac{1}{2}
\end{array}\right) m_{0}^{\prime}
$$

where, $m_{0}^{\prime}=0.05 \mathrm{eV}$. The mass eigenvalues and mixing angles of (9) are,

$$
\begin{gathered}
m_{1,2}=\frac{m_{0}^{\prime}}{2}[2-2 \epsilon-\eta \pm x], \quad m_{3}=\eta m_{0}^{\prime} ; \\
x^{2}=12 \epsilon^{2}-4 \eta \epsilon+\eta^{2} \\
\tan 2 \theta_{12}=\frac{2 \sqrt{2}}{2-\frac{\eta}{\epsilon}} ; \\
\tan ^{2} \theta_{23}=1 ; \quad \sin \theta_{13}=0 .
\end{gathered}
$$

The solar and atmospheric mass-square differences can be expressed as,

$$
\triangle m_{21}^{2}=\left[m_{2}^{2}-m_{1}^{2}\right], \quad \triangle m_{23}^{2}=\left[m_{2}^{2}-m_{3}^{2}\right] .
$$

with the latest observational bounds,

$$
\triangle m_{21}^{2}=(7.2-9.5) \times 10^{-5} \mathrm{eV}^{2}, \quad \triangle m_{23}^{2}=(1.28-4.2) \times 10^{-3} \mathrm{eV}^{2}
$$

By convention $\triangle m_{21}^{2}$ is always defined as positive, $m_{2}^{2} \geq m_{1}^{2}$, for $\tan ^{2} \theta_{12}<1$. From eq.(12), it is clear that $\tan ^{2} \theta_{12}$ can be fixed by proper choice of the flavour twister term $\frac{\eta}{\epsilon}$. 


\section{Numerical analysis and results}

Both graphical and numerical methods are employed to find the value of $\eta$ (or $\epsilon$ ) for a fixed $\tan ^{2} \theta_{12}$. We first graphically solve eqs. (12), (14), (15) using Mathematica Programmes(MATHEMATICA 5). Numerical solution of eq.(14) leads to exact values of $\eta$ (or $\epsilon$ ) consistent with $\triangle m_{21}^{2}$ and $\triangle m_{23}^{2}$ in the data (15). The process requires overall consistent approach to pindown the exact values of $\eta$ (or $\epsilon$ ) for the given $\tan ^{2} \theta_{12}$ value.

For demonstration we consider the case for $\tan ^{2} \theta_{12}=0.5$. Two different values of $\frac{\eta}{\epsilon}$ satisfying $\tan ^{2} \theta_{12}=0.5$ are $\frac{\eta}{\epsilon}=1$ and $\frac{\eta}{\epsilon}=3$. From the graphical solution of eqs. (12), (14), (15), it is found that there are two positive ranges of values of $\eta$ for $\frac{\eta}{\epsilon}=1$ and one negative range $\frac{\eta}{\epsilon}=3$ respectively, for the given $\tan ^{2} \theta_{12}$. The three allowed ranges of $\eta$ are tabulated as,

\begin{tabular}{|c|c|l|}
\hline$\frac{\eta}{\epsilon}$ & $\tan ^{2} \theta_{12}$ & range of $\eta$ \\
\hline 1 & 0.5 & $0.6603-0.6618$ \\
1 & 0.5 & $0.0048-0.0064$ \\
3 & 0.5 & $-0.0187-0.0142$ \\
\hline
\end{tabular}

We are interested only in the first range in the present analysis, as this range leads to neutrino mass model having opposite $\mathrm{CP}$ parity in the first two mass eigenvalues. The other two ranges of $\eta$ predict even $\mathrm{CP}$ parity in mass eigenvalues. For deviation from tribimaximal mixings, we also find out the ranges of $\eta$ for $\tan ^{2} \theta_{12}=0.45,0.35$ using the same procedure and results are presented in Table-1.

Table-1: Lowering of $\tan ^{2} \theta_{12} \leq 0.5$ for fixed $\tan ^{2} \theta_{23}=1, \sin \theta_{13}=0$.

\begin{tabular}{|r|c|c|c|l|}
\hline$\frac{\eta}{\epsilon}$ & $\tan ^{2} \theta_{12}$ & range of $\eta$ & $\triangle m_{21}^{2}\left(10^{-5} \mathrm{eV}^{2}\right)$ & $\triangle m_{23}^{2}\left(10^{-3} \mathrm{eV}^{2}\right)$ \\
\hline 1 & 0.5 & $0.6603-0.6618$ & $9.499-7.199$ & $1.410-1.405$ \\
0.8405 & 0.45 & $0.5865-0.5878$ & $9.500-7.200$ & $2.032-2.029$ \\
0.4462 & 0.35 & $0.3622-0.3628$ & $9.430-7.200$ & $4.010-4.008$ \\
\hline
\end{tabular}

It is seen that the quantity $\triangle m_{21}^{2}$ is quite sensitive to the corresponding change in the input values of $\eta$, whereas such variation is almost absent in the prediction of $\triangle m_{23}^{2}$.

We also present the graphical solution for the case $\tan ^{2} \theta_{12}=0.45$ along with a correlation plot between $\Delta m_{21}^{2}$ and $\Delta m_{23}^{2}$ in Fig.1. Similar diagrams and correlation plots for other two remaining cases in Table-1, can in principle be drawn in the same fashion. 

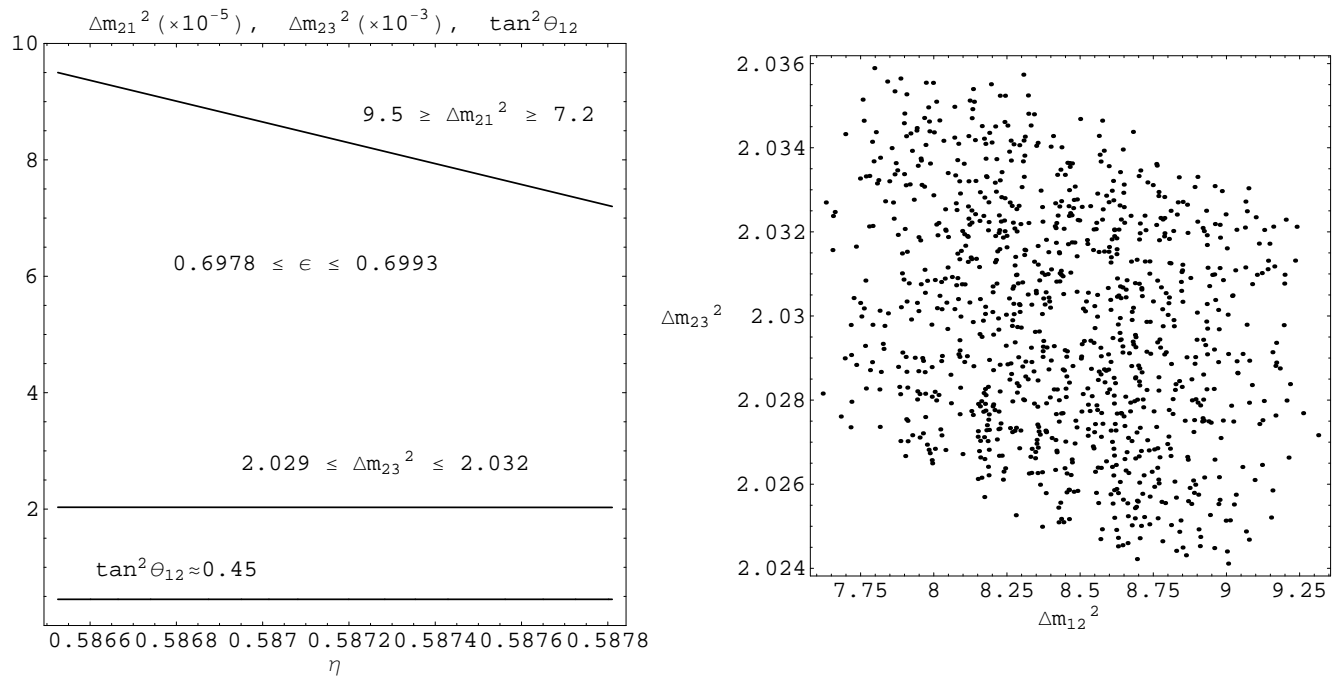

Figure 1: Predictions on $\Delta m_{21}^{2}$ in the unit $\left(10^{-5} \mathrm{eV}^{2}\right)$ and $\Delta m_{23}^{2}$ in the unit $\left(10^{-3} \mathrm{eV}^{2}\right)$ for the value $\tan ^{2} \theta_{12}=0.45$ in the range $0.5865 \leq \eta \leq 0.5878$ and the corresponding correlation graph.

For completeness, we also give other two possible solutions of flavour twister $\eta / \epsilon$ for $\tan ^{2} \theta_{12}=0.5,0.45$, which predict even CP parity in the first two mass eigenvalues in Table-2. Such models are not stable under radiative corrections in MSSM. However it is interesting to note that these two types of inverted hierarchical models have the same origin with the same form of mass matrix.

Table-2: Lowering of $\tan ^{2} \theta_{12} \leq 0.50$ for fixed $\tan ^{2} \theta_{23}=1, \sin \theta_{13}=0$ for different ranges of $\eta($ or $\epsilon)$ which lead to even CP parity in mass eigenvalues.

\begin{tabular}{|l|l|l|l|r|}
\hline$\frac{\eta}{\epsilon}$ & $\tan ^{2} \theta_{12}$ & range of $\eta$ & $\Delta m_{21}^{2}\left(10^{-5} \mathrm{eV}^{2}\right)$ & $\Delta m_{23}^{2}\left(10^{-3} \mathrm{eV}^{2}\right)$ \\
\hline 1.00 & 0.50 & $0.0048-0.0064$ & $7.200-9.499$ & $2.499-2.499$ \\
0.84 & 0.45 & $0.0040-0.0053$ & $7.173-9.499$ & $2.502-2.503$ \\
3.00 & 0.50 & $-0.0187--0.0142$ & $9.499-7.199$ & $2.630-2.595$ \\
3.16 & 0.45 & $-0.0193--0.0147$ & $9.500-7.199$ & $2.626-2.596$ \\
\hline
\end{tabular}




\section{Summary and conclusion}

The inverted hierarchical neutrino mass model characterised by opposite $\mathrm{CP}$ parity in the first two mass eigenvalues $\left(m_{1},-m_{2}, m_{3}\right)$, is generally stable under renormalization group evolution in MSSM, but its predicted value of solar mixing angle, is generally larger than the observed data. The usual approach to lower the prediction of solar mixing angle is carried out through the correction from charged lepton mass matrix. The texture of neutrino mass matrix studied in the present work, has the potential to predict lower values of solar mixing angle beyond its tribimaximal value without taking usual correction from charged lepton sector. This is carried out by changing the input value of a flavour twister present in the mass matrix[19]. The presence of a 2-3 symmetry in the mass matrix, protects the conditions for $\tan ^{2} \theta_{23}=1$ and $\sin \theta_{13}=0$.

The analysis presented here though phenomenological, supports the survival of the inverted hierarchical neutrino mass model with opposite $\mathrm{CP}$ parity in the first two mass eigenvalues. This model is not yet ruled out and this competes with the normal hierarchical model at equal footing. Future neutrino experiments $[5,20]$ are expected to confirm the correct pattern of neutrino mass hierarchy and will be capable of probing very low $\theta_{13}$. The present work may be useful to model building on tri-bimaximal mixings(TBM) and possible deviations based on discrete as well as non-abelian gauge groups[21].

\section{Acknowledgement}

One of us (NNS) is thankful to Prof. Guido Altarelli and Prof. Ernest Ma for useful interactions during WHEPP-9, held at Institute of Physics, Bhubaneswar, India during Jan,3-14, 2006. Monisa Rajkhowa acknowledges the UGC for awarding fellowship under fellowship improvement programme (FIP).

\section{References}

[1] G.Altarelli, Nucl. Phys. B. Proc. Suppl.143, 470 (2005).

[2] P. F. Harrison, D. H. Perkins, W. G. Scott, Phys.Lett.B530, 167 (2002), hep-ph/0202074. 
[3] B.Aharmim et al, (SNO Collaboration),Phys.Rev.C72,055502(2005),nucl-ex/0502021; A. Strumia and F.Vissani,Nucl. Phys.B726, 294 (2005), hep-ph/0503246.

[4] C.S.Lam, Phys.Rev.D71,093001(2005); hep-ph/0508008.

[5] A. de Gouvea and Walter Winter, Phys. Rev. D73, 033003 (2006), hep-ph/0509359.

[6] G. Altarelli, F. Feruglio, Phys. Rep.320, 295 (1999).

[7] N. Nimai Singh, M. Patgiri, M. K. Das, Pramana J.Phy.66,361 (2006).

[8] Carl H. Albright, Phys.lett. B599, 285 (2004), hep-ph/0407155.

[9] D.Indumati and M. V. N. Murthy, Phys. Rev. D71, 013001 (2005), hep-ph/0407336 S.Palomares-Ruiz, S.T. Petcov, Nucl. Phys. B712, 392 (2005), hep-ph/0406096 R. Gandhi, P. Ghoshal, S. Goswami, P. Mehta, S. Uma Sankar, hep-ph/0506145 S. Choubey, W. Rodejohann, hep-ph/0506102 A. Gouvea, W. Winter, Phys. Rev. D73, 033003 (2006), hep-ph/0509359.

[10] S. F. King and N. Nimai Singh, Nucl. Phys. B596,81 (2001).

[11] M. Patgiri and N. Nimai Singh, Phys. lett.B567(2003)69.

[12] An incomplete list, J. Ellis, S. Lola, Phys. Lett. B458,310(1999),hep-ph/9904279 N. Haba, Y. Matsui, N. Okamura, Prog. Theor. Phys. 103, 807 (2000),hep-ph/9911481; J. A. Casas, J. R. Espinosa, A. Ibarra, I. Navarro, Nucl. Phys. B556,3 (1999), hep-ph/9910420 N. Haba and N. Okamura, Euro. Phys. J.C14, 347 (2000); Anjan S. Joshipura, Phys. Lett. B543,276 (2002), hep-ph/0205038; A. S. Joshipura, S. D. Rindani, N. Nimai Singh, Nucl.Phys.B660,362 (2003); M. K. Das, M. Patgiri, N. Nimai Singh, Pramana J. Phys.65,995 (2005).

[13] R. Barbieri, L. J. Hall, D. Smith, A. Strumia and N. Weiner, JHEP, 9812:017(1998),hep-ph/9807235.

[14] K. S. Babu and R. N. Mohapatra, Phys. Lett.B532,77(2002).hep-ph/0201176. 
[15] M. Patgiri and N. Nimai Singh, IJMP,A18,443(2003); Indian J.Phys.A76,423(2002); N. Nimai Singh and M. Patgiri, IJMP,A17,3629(2002).

[16] G. Guinti, M. Tanimoto, Phys. rev. D70, 157 (2002), hep-ph/0207096 Andrea Romanino, Phys. Rev. D70, 013003 (2004), hep-ph/0402258; G. Altarelli, F. Feruglio, I. Masina, Nucl. Phys. B689, 157 (2004), hep-ph/0402155; P. H. Framptom, S. T. Petcov, W. Rodejohann, Nucl. Phys. B687, (2004) 31, hep-ph/0401206; C. A. de S. Pires, J. Phys. G30, 1329 (2004), hep-ph/0404146; J. Ferrandis, S.Pakvasa, Phys. Rev. D71, 033004 (2005); Phys.Lett. B603,184(2004); Zhi-Zhong Xing, Phys. Lett.B618, 141 (2005), hep-ph/0503200 S. Antusch, S. F. King, Phys. Lett. B631,42 (2005), hep-ph/0508044 H. Fritzsch, Zhi-Zhong Xing, Phys. Lett. B634, 514 (2006), hep-ph/0601104.

[17] Ernest Ma, Phys. Rev. D70, 031901 (2004), hep-ph/0404199.

[18] R.N.Mohapatra, S.Nasri, Hai-Bo Yu, hep-ph/0605020.

[19] Naoyuki Haba, Atsushi Watanabe, Koichi Yoshioka, Phys. Rev. Lett.97(2006)041601, hep-ph/0603116.

[20] S. K. Agarwalla, A. Raychaudhuri, A. Samanta, Phys. Lett.B629,33 (2005).

[21] E. Ma, mod. Phys. Lett. A20, 2601 (2005), hep-ph/0508099 A. Zee, Phys. Lett. B630, hep-ph/0508278; G. Altarelli, F. Feruglio, nucl. Phys. B20, 64 (2005),hep-ph/0504165, hep-ph/0512103; W. Grimus, L. Lavoura, JHEP 0601, 018(2006), hep-ph/0509239 I. de M. Varzielas, S. F. King, G. G. Ross, hep-ph/0512313 N. Haba, A. Watanabe, K. Yoshioka, hep-ph/0603116. 